\title{
On Devising Boussinesq-type Equations with Bounded Eigenspectra: Two Horizontal
} Dimensions

\section{Eskilsson, Claes; Engsig-Karup, Allan Peter}

Published in:

Proceedings of the 18th European Conference on Mathematics for Industry (ECMI 2014)

Publication date:

2015

Link back to DTU Orbit

Citation (APA):

Eskilsson, C., \& Engsig-Karup, A. P. (2015). On Devising Boussinesq-type Equations with Bounded Eigenspectra: Two Horizontal Dimensions. In Proceedings of the 18th European Conference on Mathematics for Industry (ECMI 2014) Springer.

\section{General rights}

Copyright and moral rights for the publications made accessible in the public portal are retained by the authors and/or other copyright owners and it is a condition of accessing publications that users recognise and abide by the legal requirements associated with these rights.

- Users may download and print one copy of any publication from the public portal for the purpose of private study or research.

- You may not further distribute the material or use it for any profit-making activity or commercial gain

- You may freely distribute the URL identifying the publication in the public portal

If you believe that this document breaches copyright please contact us providing details, and we will remove access to the work immediately and investigate your claim. 


\title{
On Devising Boussinesq-type Equations with Bounded Eigenspectra: Two Horizontal Dimensions
}

\author{
Claes Eskilsson and Allan P. Engsig-Karup
}

\begin{abstract}
Boussinesq-type equations are used to describe the propagation and transformation of free-surface waves in the nearshore region. The nonlinear and dispersive performance of the equations are determined by tunable parameters. Recently the authors presented conditions on the free parameters under which a Nwogu-type equations would yield bounded eigenspectra [5]. This leads to a global conditional CFL time-step restriction which is shown to not be affected by the discretisation method and in this sense the CFL condition is tamed to impose a minimal constraint. In this paper we extend the previous study and provide numerical experiments which confirms the theoretical results also is valid in two horizontal dimensions.
\end{abstract}

\section{Introduction}

In coastal engineering application the use of classical FEM for low-order spatial discretisation of wave equations has been used to describe complex geometries by using graded meshes. An immediate advantage of graded meshes is the ability to significantly decrease the total number of unknowns in the discretisation making it possible to improve the computational efficiency by spatially only resolving geometry or features of the solution where it is needed [11]. The use of flexible mesh discretisation methods is gaining more popularity since with this basis it becomes more straightforward to describe wave-structure interactions in complex settings (e.g. harbour and coastal areas), cf. recent review given in [1]. The downside for most wave models is that the use of such graded meshes may impose more severe

Claes Eskilsson

Department of Shipping and Marine Technology, Chalmers University of Technology, SE-412 96 Gothenburg, Sweden, e-mail: claes.eskilsson@ chalmers.se

Allan P. Engsig-Karup

Department of Applied Mathematics and Computer Science, Technical University of Denmark, DK-2800 Kgs. Lyngby, Denmark e-mail: apek@dtu.dk 
time-stepping restrictions due to global condition CFL stability constraints. From an algorithmic perspective remedies to this problem is found in local time-stepping algorithms, semi-implicit temporal integration, or the use of only low-order and less accurate simulation resulting from artificial dispersion and dissipation errors. Highorder discretisation have the potential to address all of these downsides, however, is known to often have operator eigenspectra which grow fast and therefore CFL conditions often become to severe for practical use. Thus, state-of-the-art techniques such as the high-order spectral element/ $h p$ method (SEM) have still not caught much interest in coastal engineering applications [6] despite that it offer good and flexible opportunities for balancing work effort and accuracy.

In this work, we consider the extension [5] and device a Boussinesq-type model in two space dimensions, which is amendable to use high-order SEM and unstructured meshes. As in [2-4], we demonstrate that graded meshes does not pose a significantly challenge for an implicitly-implicit formulations for wave-structure problems since the global CFL condition is governed by bounded eigenpectra and in this sense can be tamed [13].

\section{Boussinesq Equations}

Consider the weakly nonlinear and dispersive Boussinesq-type equations due to Nwogu [10]. In the original form, the set of equations - with unbounded operator spectrum - can be stated in two horizontal dimensions as

$$
\begin{aligned}
& \eta_{t}+\nabla \cdot(d \mathbf{u})+\varepsilon \nabla \cdot(\eta \mathbf{u})+\mu^{2} \Gamma_{20}=\mathscr{O}\left(\varepsilon^{2}, \mu^{2} \varepsilon, \mu^{4}\right), \\
& \mathbf{u}_{t}+\nabla \eta+\varepsilon(\mathbf{u} \cdot \nabla) \mathbf{u}+\mu^{2} \Delta_{20}=\mathscr{O}\left(\varepsilon^{2}, \mu^{2} \varepsilon, \mu^{4}\right),
\end{aligned}
$$

where the dispersive terms read

$$
\begin{aligned}
\Gamma_{20} & =\nabla \cdot\left[a_{1} d^{3} \nabla\left(\nabla \cdot \tilde{\mathbf{u}}+a_{2} d^{2} \nabla(\nabla \cdot(d \mathbf{u}))\right],\right. \\
\Delta_{20} & =b_{1} d^{2} \nabla\left(\nabla \cdot \mathbf{u}_{t}\right)+b_{2} d \nabla\left(\nabla \cdot\left(d \tilde{\mathbf{u}}_{t}\right)\right) .
\end{aligned}
$$

Here $\eta(\mathbf{x}, t)$ is the free surface elevation, $\mathbf{u}(\mathbf{x}, t)$ is the horizontal velocity at the reference level $z_{\alpha}$ and $d(\mathbf{x})$ is the still water depth. The constants $\left(a_{1}, a_{2}, b_{1}, b_{2}\right)$ govern the dispersive properties of the equations.

Using the enhancement technique [9] additional free parameters are introduced to the equations. This approach exploits that the Boussinesq-type equations are derived by a truncation procedure, and as long as modification are on the size of the magnitude of the truncation errors, the approach is feasible. The resulting equations read [9]:

$$
\begin{aligned}
\Gamma_{20} & =\nabla \cdot\left[a_{1} d^{3} \nabla(\nabla \cdot \mathbf{u})+\left(a_{2}-\beta_{1}\right) d^{2} \nabla(\nabla \cdot(d \mathbf{u}))-\beta_{1}\left(d^{2} \nabla \eta_{t}\right)\right], \\
\Delta_{20} & =\left(b_{1}-\alpha_{1}\right) d^{2} \nabla\left(\nabla \cdot \mathbf{u}_{t}\right)+b_{2} d \nabla\left(\nabla \cdot\left(d \mathbf{u}_{t}\right)\right)-\alpha_{1} d^{2} \nabla\left(\nabla^{2} \eta\right),
\end{aligned}
$$


where an optimum choice - with regard to linear dispersion characteristics - of the free parameters is $\left(b_{1}+b_{2}, \alpha_{1}, \beta_{1}\right)=(-0.395,0.011,0.039)$. The equations exhibit an unbounded eigenspectrum and we will hence refer to this setting as unbounded Boussinesq equations (UBE). However, as shown in [5], under certain conditions on the free parameters this set of equations will exhibit a bounded eigenspectrum. This observation was numerically supported by simulations in one horizontal dimension. Using the setting $\left(b_{1}+b_{2}, \alpha_{1}, \beta_{1}\right)=(-0.400,0,0.015)$ gives a bounded eigenspectrum while retaining good dispersion characteristics. We will refer to this setting as bounded Boussinesq equations (BBE).

\section{Numerical Discretization}

To develop a high-order spectral/hp element method [8] that works on unstructured meshes we use the Method of Lines, where a semi-discrete system of equations is formed by spatial discretisation using a Galerkin method.

We introduce approximate solutions, e.g., $u_{\delta}$ to $u$, where $u_{\delta} \in \chi$ with $\chi=\{u \mid u \in$ $\left.H^{1}(\Omega), u(\mathbf{x})=g(\mathbf{x}), \mathbf{x} \in \partial \Omega\right\}$ is the trial space and $\mathscr{V}=\left\{v \mid v \in H^{1}(\Omega), v(\mathbf{x})=0, \mathbf{x} \in\right.$ $\partial \Omega\}$ is the set of all test functions.

We partition the domain $\Omega_{h} \subset \Omega$ to obtain a tessellation $\mathscr{T}_{h}$ which consists of $N_{e l}$ non-overlapping elements $\mathscr{T}_{e}$ such that $\cup_{e=1}^{N_{e l}} \mathscr{T}_{e}=\mathscr{T}_{h}$. We approximate the solutions $(\eta, u, v, w, q) \in \mathscr{V} \subset C^{0}(\Omega)$ with piece-wise continuous $P$ 'th order polynomial approximations $\left(\eta_{\delta}, u_{\delta}, v_{\delta}, w_{\delta}, q_{\delta}\right) \in \mathscr{V}_{\delta}$ where the discrete space is given as $\mathscr{V}_{\delta}=\left\{v \in H^{1}\left(\mathscr{T}_{h}\right):\left.v\right|_{\mathscr{T}_{e}} \in \mathscr{P}^{P}\left(\mathscr{T}_{e}\right) \in \mathscr{T}_{h}\right\}$. Each element $\mathscr{T}_{e}$ is filled with $N_{P}$ local nodes.

In two space dimensions, we can represent the approximate solutions in the form of a nodal expansion

$$
\eta_{\delta}(\mathbf{x}, t)=\sum_{e=1}^{N_{e l}} \sum_{n=1}^{N_{P}} \hat{\eta}_{n}^{e}(t) l_{n}\left(\chi_{e}^{-1}(\mathbf{x})\right), \quad(\mathbf{x}) \in \mathscr{T}_{h}, \quad t \geq 0
$$

in which $\hat{\eta}_{n}^{e}(t)$ are local expansion coefficients and $l_{n}(\mathbf{x})$ is the multivariate $n$ 'th Lagrange polynomial with cardinal property $l_{n}\left(\mathbf{x}_{i}\right)=\delta_{n i}$ defined from the set of unique vertices $\left(\mathbf{x}_{i}\right)=\chi_{e}\left(\mathbf{r}_{i}\right)$ defining the local nodes on element $e . \chi_{e}: \mathbf{r} \rightarrow \mathbf{x}$ is a local affine co-ordinate mapping from a standard element $\mathscr{T}_{s t}$ to an elemental region $\mathscr{T}_{e}=\left\{x \mid x \in \chi_{e}(\mathbf{r})\right\}$. For a straight-sided reference triangle, we have $\mathscr{T}_{s t}=$ $\{\mathbf{r}=(r, s) \mid(r, s) \geq-1 ; r+s \geq 0\}$. On the standard triangular element one can use the symmetric and optimized node distribution generated by an explicit warp and blend procedure described in [12]. In a similar way, for a straight-sided reference quadrilateral, we have $\mathscr{Q}_{s t}=\{\mathbf{r}=(r, s) \mid-1 \leq(r, s) \leq 1\}$ and we use the nodes defined by a tensor product of Gauss-Lobatto-Legendre points in the two directions.

For brevity we present the method for the constant depth case. The weak formulation of (1) and (3) can be stated as 


$$
\begin{aligned}
\iint_{\mathscr{T}_{h}} v_{\delta}\left[\left(1-\mu^{2} \beta_{1} d^{2} \nabla^{2}\right) \eta_{t}\right. & +\nabla \cdot((d+\varepsilon \eta) \mathbf{u}) \\
& \left.+\mu^{2}\left(\alpha+\frac{1}{3}-\beta_{1}\right) d^{3} \nabla^{2} w\right] d \mathbf{x}=0, \\
\iint_{\mathscr{T}_{h}} v_{\delta}\left[\mathbf{u}_{t}+\mu^{2}\left(\alpha-\alpha_{1}\right) d^{2} \nabla w_{t}\right. & \left.+\nabla \eta+\varepsilon(\mathbf{u} \cdot \nabla) \mathbf{u}-\mu^{2} \nabla q\right] d \mathbf{x}=0
\end{aligned}
$$

where $\alpha=b_{1}+b_{2}$ and having neglected all $\mathscr{O}\left(\varepsilon^{2}, \mu^{2} \varepsilon, \mu^{4}\right)$-terms. Further, we have introduced the auxiliary variables $w=\nabla \cdot \mathbf{u}$ and $q=\alpha_{1} d^{2}\left(\nabla^{2} \eta\right)$ to resolve the thirdorder spatial derivatives Applying the Divergence theorem gives

$$
\begin{gathered}
\iint_{\mathscr{T}_{h}}\left[\left(v_{\delta}+\mu^{2} \beta_{1} d^{2} \nabla v_{\delta} \nabla\right) \eta_{t}-\nabla v_{\delta} \cdot((d+\varepsilon \eta) \mathbf{u})\right. \\
\left.-\mu^{2}\left(\alpha+\frac{1}{3}-\beta_{1}\right) d^{3} \nabla v_{\delta} \nabla w\right] d \mathbf{x}=0 \\
\iint_{\mathscr{T}_{h}}\left[v_{\delta} \mathbf{u}_{t}+\mu^{2}\left(\alpha-\alpha_{1}\right) d^{2} v_{\delta} \nabla w_{t}+v_{\delta} \nabla \eta+\varepsilon v_{\delta}(\mathbf{u} \cdot \nabla) \mathbf{u}-\mu^{2} v_{\delta} \nabla q\right] d \mathbf{x}=0
\end{gathered}
$$

and

$$
\begin{aligned}
\iint_{\mathscr{T}_{h}} v_{\delta} w d \mathbf{x} & =\iint_{\mathscr{T}_{h}}\left[-\left(\nabla v_{\delta} \cdot \mathbf{u}\right)\right] d \mathbf{x} \\
\iint_{\mathscr{T}_{h}} v_{\delta} q d \mathbf{x} & =\iint_{\mathscr{T}_{h}} v_{\delta}\left[-\alpha_{1} d^{2}\left(\nabla v_{\delta} \cdot \nabla \eta\right)\right] d \mathbf{x},
\end{aligned}
$$

where all arising boundary terms have been omitted as we in this study are only concerned with periodic and impermeable vertical wall boundaries. For periodic domains the boundary terms naturally cancels out. For the slip wall condition the zero net flux conditions in the normal direction of the walls corresponds to

$$
\mathbf{u} \cdot \mathbf{n}=0, \quad \nabla \eta \cdot \mathbf{n}=0 \quad \nabla w \cdot \mathbf{n}=0, \quad \mathbf{x} \in \partial \Omega,
$$

giving that all arising boundary terms are zero.

In the simulations presented we integrate in time using the explicit third-order Adams-Bashforth scheme. The resulting linear system is solved with GMRES with an ILU preconditioner. We note that it is well-known that equal-order simulations give rise to stability problems. However, rather than using different order for the free surface and the velocity variables we here apply a weak exponential filter [7] on interior bubble modes, if needed, in order to stabilize the solution.

\section{Eigenvalue Analysis}

Writing the equations in semi-discrete form $\mathbf{A} \partial_{t} \mathbf{U}=\mathbf{B} \mathbf{U}$ we are interested in the eigenvalues, $\lambda_{i}$, of the operator $\mathbf{A}^{-1} \mathbf{B}$, in order to understand the stability of the 
scheme. In Fig. 1 we present numerically obtained maximum eigenvalues as function of the polynomial order. The eigenvalues are purely imaginary and, as expected, the BBE equations remain bounded while the UBE equations grows as $P^{2}$. It is stressed that the magnitude of the bounded eigenvalues do not depend on the numerical discretization, but is a property of the governing equations. This is illustrated in Fig. 1 where the magnitude of the eigenvalue for the BBE is the same on both periodic structured quadrilaterals and unstructured triangles with wall boundaries. Please note that the computed eigenvalues for the BBE coincide with the analytic value given in [5].

(a)

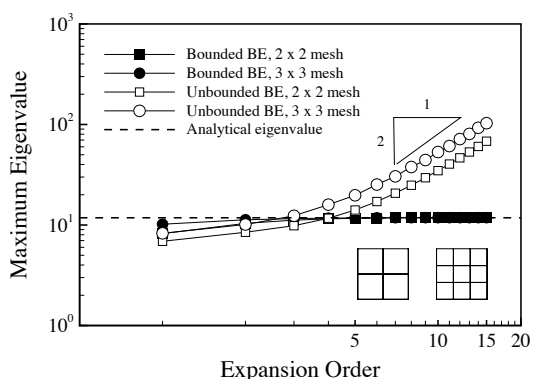

(b)

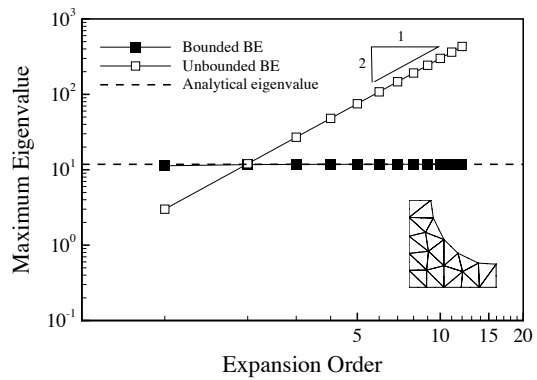

Fig. 1 Numerically observed eigenvalues. (a) Structured quadrilateral elements with periodic boundaries and (b) unstructured triangular elements with wall boundaries.

\section{Numerical Example}

A frequently used test case for Boussinesq-type equations is the run-up of a solitary wave on a cylinder. This case highlights the typical situation where the geometry are represented with sufficient accuracy by generating an unstructured mesh with a significantly higher mesh density around structures and topological features.

The size of the wave tank is $[x, y] \in[-33,25] \times[-19.2,19.2] \mathrm{m}$ with a depth of 1 $\mathrm{m}$. We utilize the symmetry of the set-up and simulate only the lower half of the domain. All boundaries are treated as wall/symmetry boundaries. The computational domain is decomposed into two different meshes with (a) 269 and (b) 116 triangular elements, see Fig. 2. Both meshes resolve the cylinder with approximately the same number of boundary edges.

The initial condition is given by Laitone's first order solitary wave solution, centered at $x=-17 \mathrm{~m}$, and we integrate the solution for $12.5 \mathrm{~s}$. Figure 3 shows the simulated run-up and subsequent scattering of the solitary wave. For mesh A we need $P \geq 5$ and for mesh B we need $P \geq 7$ in order to get acceptable results. 
(a)

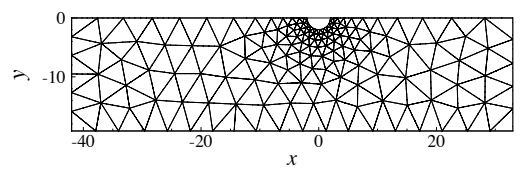

(b)

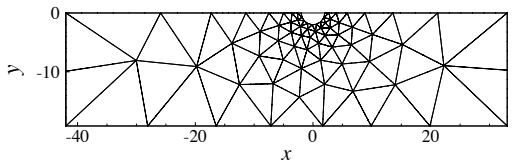

Fig. 2 Illustration of used meshes: (a) 269 elements (Mesh A) and (b) 116 elements (Mesh B).

(a)

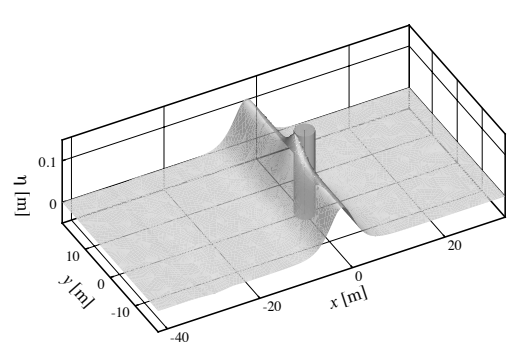

(c)

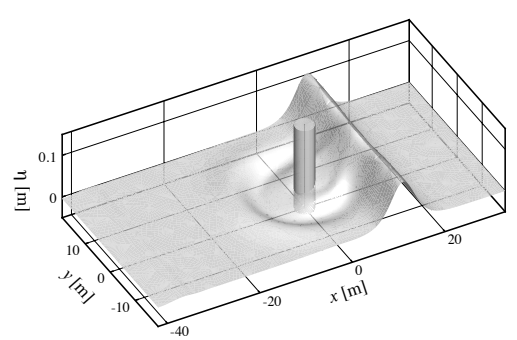

(b)

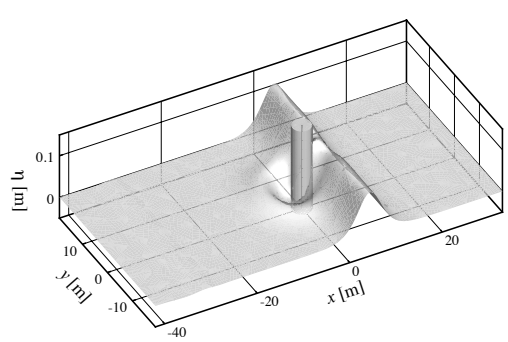

(d)

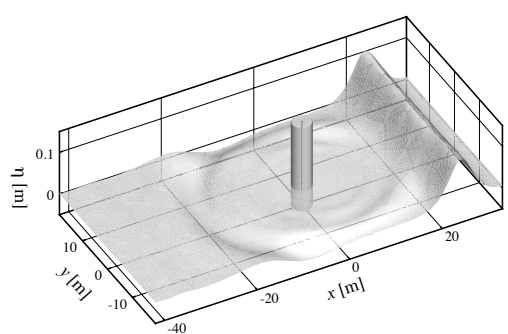

Fig. 3 Solitary wave impinging on a vertical cylinder. Simulation using mesh A with $P=6$. At time: (a) $4.5 \mathrm{~s}$, (b) $6.5 \mathrm{~s}$, (c) $8.5 \mathrm{~s}$ and (d) $12.5 \mathrm{~s}$.

In Table 1 we present the influence of the polynomial order on the maximum allowed time step. Again, a novel feature is that the maximum stable time step for BBE simulations are not dependent on the choice of $h$ and $p$. Thus it is possible to have large differences in element size as illustrated by mesh B. As a result, from Table 1 it can be seen that for practical computations it is possible to obtain significant speed up by taking advantage of high-order elements compared to standard BE settings without compromising accuracy. 
Table 1 Maximum allowed time step (in sec.) for a solitary wave impinging on a cylinder.

\begin{tabular}{lcccc}
\hline & & BBE & UBE & Rel. Diff. \\
\hline \multirow{3}{*}{ Mesh A } & $P=4$ & $6.25 \mathrm{E}-02$ & $5.21 \mathrm{E}-02$ & 1.20 \\
& $P=5$ & $6.25 \mathrm{E}-02$ & $4.03 \mathrm{E}-02$ & 1.55 \\
& $P=6$ & $6.25 \mathrm{E}-02$ & $3.21 \mathrm{E}-02$ & 1.95 \\
\hline \multirow{3}{*}{ Mesh B } & $P=6$ & $6.25 \mathrm{E}-02$ & $3.68 \mathrm{E}-02$ & 1.70 \\
& $P=7$ & $6.25 \mathrm{E}-02$ & $3.05 \mathrm{E}-02$ & 2.05 \\
& $P=8$ & $6.25 \mathrm{E}-02$ & $2.50 \mathrm{E}-02$ & 2.50 \\
\hline
\end{tabular}

\section{Conclusion}

We have analysed and demonstrated that an implicitly-implicit formulation of a Nwogu-type equation in two spatial dimensions can be made accurate and efficient without compromising robustness by using unstructured high-order spectral element/hp methods. This work opens the road towards robust mesh-adaptive solutions considered in ongoing work.

\section{References}

1. Brocchini, M.: A reasoned overview on Boussinesq-type models: the interplay between physics, mathematics and numerics. Proc. R. Soc. Lond. A 469(2160) (2013)

2. Engsig-Karup, A., Hesthaven, J., Bingham, H., Madsen, P.: Nodal DG-FEM solutions of highorder Boussinesq-type equations. J. Engineering Math. 56, 351-370 (2006)

3. Engsig-Karup, A., Hesthaven, J., Bingham, H., Warburton, T.: DG-FEM solution for nonlinear wave-structure interaction using boussinesq-type equations. Coastal Engineering 55, 197-208 (2008)

4. Engsig-Karup, A.P.: Unstructured nodal DG-FEM solution of high-order boussinesq-type equations. Ph.D. thesis, PhD. Thesis. Department of Mechanical Engineering, Technical University of Denmark (2006)

5. Eskilsson, C., Engsig-Karup, A.P.: On devising Boussinesq-type models with bounded eigenspectra: One horizontal dimension. Journal of Computational Physics 271, 261-280 (2014)

6. Eskilsson, C., Engsig-Karup, A.P., Sherwin, S.J., Hesthaven, J.S., Bergdahl, L.: The next step in coastal numerical models: spectral/hp element methods? In: Proceedings of the WAVES2005 Conference, Madrid, 712 July 2005 (2005)

7. Hesthaven, J.S., Warburton, T.: Nodal Discontinuous Galerkin Methods: Algorithms, Analysis, and Applications. Springer (2008)

8. Karniadakis, G., Sherwin, S.: Spectral/hp Element Methods for Computational Fluid Dynamics: Second Edition. Numerical Mathematics and Scientific Computation. Oxford (2005)

9. Madsen, P., Schäffer, H.A.: Higher order Boussinesq-type equation for surface gravity waves - derivation and analysis. Proc. R. Soc. Lond. A 356, 3123-3181 (1998)

10. Nwogu, O.: Alternative form of Boussinesq equations for nearshore wave propagation. J. Waterway, Port, Coastal and Ocean Eng., ASCE 6(119), 618-638 (1993)

11. Sørensen, O.R., Schäffer, H.A., Sørensen, L.S.: Boussinesq-type modelling using an unstructured finite element technique. Coastal Engineering 50, 181-198 (2004)

12. Warburton, T.: An explicit construction for interpolation nodes on the simplex. J. Engineering Math. 56(3), 247-262 (2006)

13. Warburton, T., Hagstrom, T.: Taming the CFL number for discontinuous Galerkin methods on structured meshes. SIAM J. Numer. Anal. 46(6), 3151-3180 (2008) 